\title{
Nano-scale Design of New Catalysts Based on Fundamental Insight Into Surface Reactivity at the Atomic Scale
}

Flemming Besenbacher

Interdisciplinary Nanoscience Center (iNANO), University of Aarhus, DK-8000 Aarhus, Denmark, E-mail: fbe@,inano.dk, WEB: www.inano.dk/spm

For decades, single-crystal surfaces have been studied under ultra-high vacuum (UHV) conditions as model systems for elementary surface processes underlying phenomena such as heterogeneous catalysis, epitaxial growth, corrosion etc. The "surface science approach" has contributed substantially to our understanding of the processes involved in catalysis [1]. Scanning tunneling microscopy (STM) has by now been established as an outstanding and versatile technique for direct real space studies of matter at the atomic level. The recent progress in STM studies of model systems relevant to heterogeneous catalysis has shown that fundamental issues related to catalytic processes can be resolved by imaging, for example catalytically active surfaces and nanostructures with atomic resolution [2-4]. In this talk I will discuss selected examples from my own research group who has used the unique capabilities of our high-resolution, fast-scanning STM to reveal fundamental processes of model catalysts. I will illustrate the coming of a new era in the sense that the insight gained from studying model systems has advanced to a stage where the insight and ideas may lead to nano-scale design of new and improved high surface area catalysts operating under realistic conditions [5-8].

[1] F. Besenbacher et al., Science 279 (1998) 1913

[2] R. Schaub et al., Science 299 (2003) 377

[3] E. Wahlström et al., Science 303 (2004) 511

[4] E. Wahlström et al., Phys. Rev. Lett. 90 (2003) 026101

[5] S. Helveg et al Phys Rev. Lett. 84 (2000) 951

[6] J. V. Lauritsen et.al., J. Catal. 197 (2001) 1

[7] J.V. Lauritsen et al., Nanotech, 14 (2003) 385

[8] R.T. Vang, et al., Nature Materials 4 (2005) 160 